\title{
Condições socioecológicas familiares nos primeiros dois anos de vida de crianças quilombolas no Pará: um estudo de base populacional
}

\author{
Family socioecological conditions in the first two years of quilombola children in Pará: \\ a population-based studyl \\ Raissa Cecília Rosalino Guimarãesa , Hilton Pereira da Silva ${ }^{b}$, Edson Marcos Leal Soares Ramosc \\ a Nutricionista. Mestre em Saúde Coletiva. Universidade Federal do Pará. \\ 'Médico e Biólogo. Doutor em Antropologia Universidade Federal do Pará. \\ Estatístico. Doutor em Engenharia de Produção. Universidade Federal do Pará.
}

RESUMO

Objetivo: Esta pesquisa analisou a situação de crescimento de crianças de 22 comunidades remanescentes de quilombos em diversos ecossistemas do Estado do Pará e a relação com as condições socioecológicas familiares.

Materiais e Métodos: Trata-se de um estudo observacional, transversal, com amostra obtida por conveniência e demanda espontânea, constituída por crianças entre 0 e 24 meses de idade, de ambos os sexos, e seus pais ou responsáveis. Para análise utilizou-se medidas antropométricas das crianças e entrevistas semiestruturadas sobre aspectos socioeconômicos, hábitos de vida e saneamento ambiental com cada chefe de família. Os índices Peso-para-Idade, Estatura-para-Idade e Índice de Massa Corporal-para-Idade foram os descritores do estado nutricional das crianças. Para este estudo foram considerados como variáveis dependentes os dados antropométricos e como variáveis independentes os fatores socioecológicos, analisados por meio do teste de Qui-quadrado e Análise de Correspondência.

Resultados: A amostra foi constituída por 110 crianças e 87 adultos. O estudo mostrou que as comunidades investigadas não possuem formas adequadas de destinação do esgoto e das fezes, e a água utilizada nos domicílios é majoritariamente proveniente de poços abertos, evidenciando precária infraestrutura, dificuldade de acesso à água potável e saneamento ambiental inexistente. A análise antropométrica demonstra ocorrência considerável de indivíduos com baixo peso $(6,8 \%)$ e déficit estatural $(23,4 \%)$, ao mesmo tempo em que se identifica excesso de peso em algumas crianças $(11,1 \%)$.

Conclusão: Os dados mostram uma precária infraestrutura familiar e um processo de transição nutricional precoce entre os quilombolas, e reforçam a importância da realização de pesquisas com delineamento transdisciplinar.

Palavras-chave: saúde da população negra; Amazônia; transição nutricional; crescimento; quilombo.

\begin{abstract}
Objective: This study analyzed the patterns of growth of children from 22 Quilombola communities from several ecosystems in the State of Pará, in relation to the socio-ecological conditions of their families.

Material and Methods: This is an observational, cross-sectional study, with convenience sampling by spontaneous demand of the communities that consisted of children between 0 and 24 months of age, of both sexes, and their parents or guardians. Data collection included anthropometric evaluation of the children, and the application of a semi-structured questionnaire to the heads of household about socio-economic conditions, life habits, and environmental health. Weight-for-age, Height-for-age and Body Mass Index-for-age were evaluated. The anthropometric data of children were included as the dependent variables and the socio-ecologic factors configured the independent variables. Chi-square Test and Correspondence Analysis were performed.

Results: The sample consisted of 110 infants and 87 adults. The study showed that the communities investigated did not have adequate destination for waste and feces, and the water used in the houses comes mainly from open wells, demonstrating overall poor infrastructure, difficult access to potable water and non-existent environmental sanitation. Analysis of the nutritional status indicated that there was a considerable number of underweight $(6.8 \%)$ and stunted children $(23.4 \%)$ while there were also some overweight infants (11.1\%).

Conclusion: The data show precarious family infrastructure, a precocious process of nutritional transition among the Quilombola, and reinforce the importance of conducting transdisciplinary investigations.

Keywords: health of the black population, Amazon, nutrition transition, growth, quilombo.
\end{abstract}

\section{Correspondência:}

RaISSA CeCília Rosalino GUimarães

Av. Almirante Barroso, 2374, casa 3

66093-020 Belém, PA, Brasil

E-mail: raissa_guimaraes28@hotmail.com 


\section{INTRODUÇÃO}

A situação de saúde da população negra no Brasil resulta de diversos aspectos relacionados às condições sociais, econômicas, políticas e culturais de sua trajetória histórica, e reflete a fragilidade das políticas públicas criadas para atender as suas necessidades ${ }^{1,2}$. Dentro deste grupo populacional se destaca especialmente a vulnerabilidade das comunidades remanescentes de quilombos ${ }^{3-5}$.

Dentre as mazelas que acometem as populações quilombolas, os elevados índices de morbidade e mortalidade infantil continuam entre os mais graves estando, sobretudo, relacionados ao ambiente no qual as comunidades estão inseridas, caracterizado, geralmente, pela falta de infraestrutura, saneamento ambiental, inacessibilidade aos serviços de saúde e educação, e baixa condição socioeconômica ${ }^{6,7}$. Tal precariedade de condições de vida impacta diretamente a saúde das crianças, influenciando os processos de desenvolvimento, crescimento e aprendizagem, com repercussões na vida adulta ${ }^{8-10}$.

O crescimento linear é um dos melhores indicadores para se avaliar as alterações nutricionais infantis. Durante a infância, em especial nos primeiros mil dias, ou seja, da concepção aos dois anos de vida, há uma intensa proliferação de células responsáveis pelo crescimento e desenvolvimento de diversos órgãos importantes, como fígado, cérebro, ossos e músculos. Sendo assim, a falta de nutrientes essenciais, o desmame precoce, as doenças e situações ambientais adversas como pobreza, violência, opressão religiosa e étnica, entre outros, afetam o crescimento estatural e o desenvolvimento psicomotor, favorecendo o aparecimento de doenças crônicas nas fases subsequentes ${ }^{8-13}$.

A avaliação antropométrica constitui-se em importante ferramenta epidemiológica para a análise do crescimento das crianças. O acompanhamento antropométrico sistemático permite a identificação de indivíduos com maior risco de morbimortalidade, por meio da sinalização precoce da subnutrição e/ou da obesidade ${ }^{12,14}$. Diversas pesquisas têm apontado o impacto da atenção especial nos primeiros mil dias de vida, mostrando que a atenção primária adequada é fundamental para a resolução ou, pelo menos, redução dos danos à infância e ao longo da vida ${ }^{11,13}$.

O relatório de 2008 do Sistema de Vigilância Alimentar e Nutricional (SISVAN) revelou que nas famílias beneficiárias do Programa Bolsa Família há elevada prevalência de crianças com baixo Peso-para-Idade (P/I) e baixa Estaturapara-Idade $(\mathrm{E} / \mathrm{l})^{3}$. O Estado do Pará superou a média nacional $(7,9 \%)$ e da região norte $(11,7 \%)$, apresentando $13,1 \%$ de crianças com baixo P/I (indicativo de subnutrição aguda), assim como elevada prevalência de crianças com baixa estatura $(27,8 \%)$ (indicativo de subnutrição crônica), também superando a média nacional e a regional ${ }^{3}$. A última Chamada Nutricional Quilombola mostrou dados alarmantes quanto à prevalência de formas crônicas de desnutrição, em que $11,6 \%$ das crianças menores de cinco anos de grupos rurais afrodescendentes do país apresentaram déficits de crescimento ${ }^{15}$. Estudos sobre o Pará e o Brasil demonstram que grande parte das famílias quilombolas é beneficiária do Programa Bolsa Família ${ }^{3,16}$.

$\mathrm{Em}$ anos recentes verifica-se uma nova tendência no cenário epidemiológico brasileiro em que há um declínio de crianças com baixo P/I e aumento de casos de excesso de peso entre os menores de cinco anos de idade. $\mathrm{O}$ aumento da prevalência da obesidade em regiões e/ou grupos sociais submetidos à situação de insegurança alimentar e nutricional tem sido objeto de diversos estudos, mostrando que determinados fatores biossociais e econômicos estão predominantemente associados à sua etiologia ${ }^{17-19}$.

Já está bem definida a relação entre os agravos nutricionais na vida fetal ou na primeira infância, a pobreza e o desenvolvimento de obesidade e outras doenças crônicas na vida adulta. As alterações metabólicas estruturais e/ou funcionais no organismo no início da vida favorecerão o acúmulo de gordura na fase adulta, sendo as doenças associadas a este acúmulo, que estão entre as principais causas de morte na atualidade, sequelas tardias da desnutrição energético-proteica infantil ${ }^{20,21}$.

Considerando que ainda são poucos os estudos sobre a saúde de crianças quilombolas na Amazônia, o objetivo deste artigo é analisar o crescimento de crianças entre zero e dois anos de idade e a sua relação com as condições socioeconômicas familiares em um amplo conjunto de comunidades no Estado do Pará.

\section{MATERIAIS E MÉTODOS}

Trata-se de um estudo observacional, transversal, de base populacional, cuja amostra foi obtida por conveniência, com demanda espontânea nas comunidades. A população prioritária foi constituída por crianças entre 0 e 24 meses de idade, de ambos os sexos e seus pais ou responsáveis.

As comunidades remanescentes de quilombo participantes foram: Santo Antônio, localizada no município de Concórdia do Pará; África e Laranjituba, no município de Abaetetuba; Mangueiras, no município de Salvaterra; Mola, Itabatinga, Muturacá de Cima, Itapocú, Itabatinga Médio, Tomázia, Frade, Juaba, Bonfim, Taxizal e Laguinho, no município de Cametá; Abuí, Cachoeira Porteira, Tapagem, Juquirizinho, Erepecu e Arancuan, em Oriximiná e Abacatal, no município de Ananindeua (Figura 1). As áreas investigadas foram escolhidas por apresentarem histórias de 
formação, condições ecológicas, sociosanitárias e situações fundiárias distintas, cobrindo uma ampla gama de territórios, representando as diversas comunidades quilombolas no estado do Pará e os ecossistemas nos quais estas vivem. As características históricas e socioecológicas das comunidades participantes foram descritas em publicações anteriores ${ }^{22-28}$.

As informações foram coletadas em várias etapas de campo entre 2008 e 2015, em projetos contínuos - “Determinantes Socioecológicos e Biológicos da Obesidade, Diabetes e Hipertensão Arterial em Populações Afrodescendentes da Amazônia: buscando respostas concretas para a ontogenia de doenças complexas" e Projeto "Corpo Presente: representações entre quilombolas e políticas públicas", com o objetivo de investigar os efeitos dos diferentes meio ambientes, estilos de vida, mudanças socioecológicas e aspectos biomédicos relacionados à emergência de doenças agudas e crônicas entre grupos remanescentes de quilombos vivendo em diversos ecossistemas da região Amazônica ${ }^{19,23-28}$.

Para a coleta de dados utilizou-se um questionário de visita domiciliar aplicado ao/a chefe de família em cada casa visitada. As variáveis analisadas foram: (i) Sexo, (ii) Estado Civil, (iii) Nível de escolaridade, (iv) Renda familiar, (v) Recebimento de auxílio do governo, (vi) Situação do imóvel, (vii) Número de pessoas que vivem permanentemente na casa, (viii) Acesso a eletricidade, (ix) Bens de consumo da casa, (x) Situação da cozinha, (xi) Situação do banheiro, (xii) Situação do sanitário, (xiii) Acesso a água potável, (xiv) Acesso e destino do esgoto, (xv) Destino do lixo, (xvi) Uso de tabaco, (xvii) Uso de álcool, consideradas as variáveis de exposição. Estas foram categorizadas para verificar a existência de dependência entre o estado nutricional das crianças e cada uma delas.

As medidas antropométricas foram coletadas de acordo com os procedimentos internacionalmente recomendados pela Organização Mundial de Saúde ${ }^{14}$ e realizadas de maneira a reduzir o erro inter-observador. $\mathrm{O}$ peso foi aferido em quilogramas $(\mathrm{kg})$, utilizando-se uma balança digital da marca $\mathrm{WISO}^{\circledR}$, com as crianças sem sapatos e com roupas leves, e a estatura foi medida em centímetros, obtida com antropômetro marca $\mathrm{GPM}{ }^{\circledR}$, com precisão de $0,1 \mathrm{~cm}$.

Para a avaliação das crianças foram utilizados os índices Peso-para-Idade (P/I), Estatura-para-Idade (E/I) e Índice de Massa Corporal-para-Idade (IMC/I). O IMC foi calculado a partir da divisão do peso em quilogramas pela estatura em metros, elevado ao quadrado. Os valores do IMC foram classificados de acordo com o padrão de referência para crianças e os pontos de corte utilizados foram os preconizados pela Organização Mundial de Saúde ${ }^{29}$.

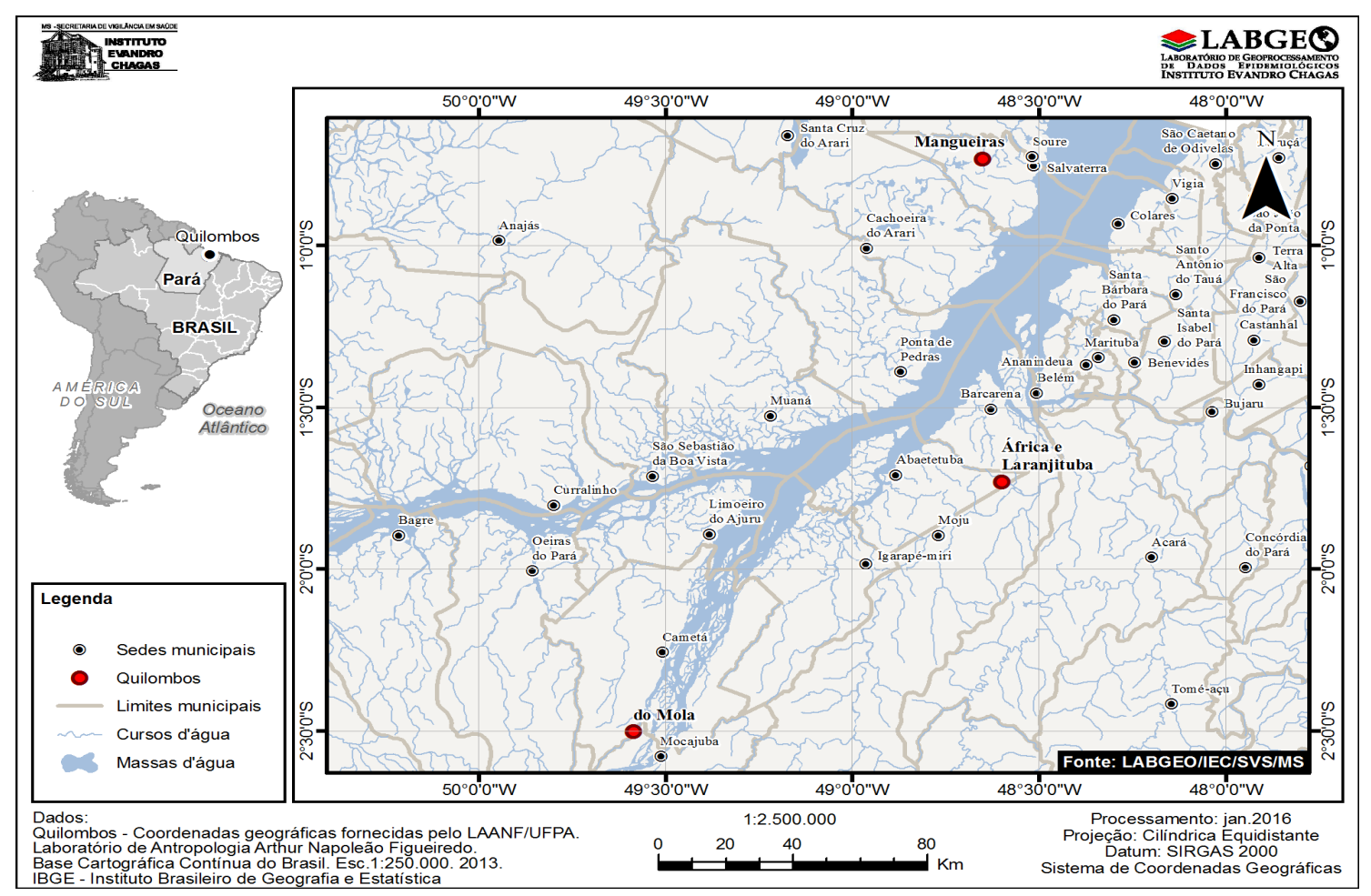

Fonte: Instituto Evandro Chagas, 2015.

Figura 1. Mapa das comunidades quilombolas de África, Laranjituba, Abaetetuba; Mola, Cametá e Mangueiras, Salvaterra, Pará. 
Os dados antropométricos foram organizados em um banco de dados no programa Microsoft Office Excel 2013, analisados e processados por meio do programa WHO Anthro Plus ${ }^{30}$. Os valores antropométricos referentes a peso e estatura foram convertidos em escores- $z$, gerando um conjunto de curvas de crescimento para os índices E/I, P/I e IMC/I que formam os descritores de estado nutricional da população amostrada. Os valores de escore-z $<-2$ para os índices E/I, P/I e IMC/I foram utilizados para caracterizar baixo peso para a idade, baixa estatura para a idade, e magreza, respectivamente. $\mathrm{O}$ valor de escore- $z>+2$ para o índice $\mathrm{IMC} / \mathrm{I}$ foi considerado como indicativo de excesso de peso ${ }^{29}$.

Os dados socioeconômicos, de moradia e saneamento básico foram armazenados e analisados no programa Statistical Package for the Social Sciences (SPSS ${ }^{\circledR}$ ) versão 24.0, sendo as características gerais das famílias descritas por distribuição de frequências simples.

Para este estudo foi considerado como desfecho o valor de escore-z $<-2$ e $>+2$ (variável dependente) para os índices E/I, P/I e IMC/I de acordo com as curvas de crescimento da OMS e as exposições analisadas se configuraram como variáveis independentes.

Considerando os diversos fatores que podem se correlacionar com o estado nutricional, foi realizada a Análise de Correspondência, uma técnica estatística multivariada exploratória de interdependência, utilizada para verificar associações entre as variáveis qualitativas ou contínuas. Primeiramente, foi realizado o teste do qui-quadrado $\left(\chi^{2}\right)$ para verificar a existência de dependência entre as variáveis. Uma vez comprovada a dependência, calculou-se o critério $\beta$. Por fim, calculou-se o coeficiente de confiança, com a finalidade de saber a probabilidade de uma categoria estar associada à outra, a partir de um procedimento baseado nos resíduos (sendo definido pela diferença entre as frequências esperadas e as observadas), por meio do qual é possível determinar a probabilidade de associação entre as categorias das variáveis, sendo classificadas em fortemente significativas ou moderadamente significativas. A análise de correspondência foi realizada com o auxílio do aplicativo Statistica ${ }^{\circledR}$, versão 6.0.

O presente estudo utilizou dados coletados em projetos aprovados pelo Comitê de Ética em Pesquisa do Instituto de Ciências da Saúde da Universidade Federal do Pará (Parecer no 499.920 e Parecer no 060/07). As comunidades e todos os participantes foram informados quanto aos seus objetivos, metodologias e resultados esperados. Todas as famílias das comunidades foram convidadas a participar de forma voluntária, sendo solicitada a participação das pessoas adultas responsáveis pelos domicílios e dos pais ou responsáveis para a participação das crianças. O interesse na pesquisa foi confirmado por meio da assinatura de um Termo de Consentimento Livre e Esclarecido (TCLE), seguindo os parâmetros das Resoluções no 196/1996 e no 466/2012 do Conselho Nacional de Saúde.

\section{RESULTADOS}

Foram analisadas inicialmente 120 crianças, no entanto, 10 crianças foram excluídas das análises dos índices E/I e IMC/I pela impossibilidade da aferição de suas estaturas. A amostra final para as análises antropométricas foi constituída por 110 indivíduos com idades entre 0 e 24 meses, representando, pelo menos, $60 \%$ das crianças nesta faixa etária das comunidades selecionadas para a pesquisa. Simultaneamente a análise das crianças, foram realizadas entrevistas com 87 pais/responsáveis.

Em relação às variáveis socioeconômicas, as entrevistas com adultos, mostraram que a maioria dos pais ou responsáveis possuía apenas o ensino fundamental incompleto $(60,9 \%)$, a renda familiar era de até dois salários mínimos para 46,0\% deles e $82,8 \%$ das famílias recebia auxílio do Programa Bolsa Família. O número médio de pessoas vivendo permanentemente em cada domicílio foi de cinco, sendo que a maioria das casas possuía entre 4 e 5 residentes. A iluminação doméstica era proveniente de gerador a diesel para $72,4 \%$. Dentre a lista de bens de consumo presentes na casa, os itens mais referidos foram: fogão, televisão, bicicleta, geladeira, aparelho de DVD, telefone celular e rádio (Tabela 1).

Em relação ao saneamento ambiental, $88,5 \%$ dos domicílios possuía cozinha interna, porém a maioria não possuía banheiro e nem sanitário interno, sendo a localização deste majoritariamente fora da casa, em média a mais de cinco metros de distância. A água utilizada para beber e para uso doméstico era proveniente de poço aberto para a maioria das famílias, sendo que 57,5\% utilizavam algum tratamento na água para beber, sendo o mais citado a adição de hipoclorito de sódio. Em relação ao destino do esgoto e das fezes, a maioria dos entrevistados respondeu que os dejetos iam para uma fossa doméstica escavada no solo, e quanto ao destino do lixo, a quase totalidade dos entrevistados respondeu que enterrava em sua propriedade. Sobre os hábitos individuais, $86,2 \%$ das pessoas entrevistadas se declarou não fumante e $24,1 \%$ dos chefes de família informou que ingere algum tipo de bebida alcoólica pelo menos uma vez na semana (Tabela $\mathbf{1}$ ).

Verificou-se que houve prevalência elevada de crianças com déficit estatural e baixo P/I (Tabela 2). Observou-se, também, alguns casos de crianças com excesso de peso, embora a maior parte das crianças estudadas estivesse eutrófica quanto ao IMC/I (Tabela 2). 
Tabela 1. Distribuição de crianças (\%) segundo características socioeconômicas.

\begin{tabular}{|c|c|c|c|}
\hline Variáveis & $\begin{array}{c}\text { Total } \\
(n=120)\end{array}$ & Variáveis & $\begin{array}{c}\text { Total } \\
(n=120)\end{array}$ \\
\hline Escolaridade do chefe de família & & Banheiro interno & \\
\hline Não Alfabetizado & 13,8 & Sim & 21,8 \\
\hline Alfabetizado & 14,9 & Não & 78,2 \\
\hline Ensino Fundamental Incompleto & 60,9 & Sanitário interno & \\
\hline Ensino Fundamental Completo & 5,7 & Sim & 28,7 \\
\hline Ensino Médio Incompleto & 3,4 & Não & 71,3 \\
\hline Ensino Superior Completo & 1,3 & Tipo de água para consumo próprio & \\
\hline Renda familiar em Salário Mínimo & & Rio e/ou igarapé & 20,7 \\
\hline$<1$ & 17,2 & Poço aberto & 48,3 \\
\hline 1 & 18,4 & Poço fechado com bomba & 12,6 \\
\hline 1,5 & 8,1 & Encanamento & 3,4 \\
\hline 2 & 46,0 & Outros & 15,0 \\
\hline 3 & 1,1 & Tipo de água para uso doméstico & \\
\hline 4 & 2,3 & Rio e/ou igarapé & 20,7 \\
\hline 5 & 4,6 & Poço aberto & 47,1 \\
\hline$>5$ & 2,3 & Poço fechado com bomba & 12,6 \\
\hline Auxílio do governo & & Encanamento & 18,5 \\
\hline Sim & 82,8 & Outros & 1,1 \\
\hline Não & 17,2 & Uso de tratamento da água para consumo próprio & \\
\hline Número de pessoas que vivem permanentemente na casa & & Sim & 57,5 \\
\hline $1-3$ pessoas & 23,4 & Não & 42,5 \\
\hline 4-5 pessoas & 27,6 & Destino do esgoto caseiro & \\
\hline 6-7 pessoas & 23,4 & Rio e/ou igarapé & 12,6 \\
\hline$>7$ pessoas & 25,6 & Céu aberto & 26,4 \\
\hline Iluminação & & Fossa & 48,4 \\
\hline Elétrica por gerador local & 72,5 & Outros & 12,6 \\
\hline Elétrica da Celpa & 13,8 & Destino do esgoto do banheiro & \\
\hline Lampião a gás & 12,6 & Rio e/ou igarapé & 12,6 \\
\hline Outros & 1,1 & Céu aberto & 17,2 \\
\hline Bens de Consumo & & Fossa & 36,9 \\
\hline Fogão & 88,5 & Outros & 33,3 \\
\hline Televisão & 74,7 & Destino do lixo & \\
\hline Bicicleta & 47,1 & Enterrado & 92,1 \\
\hline Geladeira & 41,4 & Jogado no mato & 3,4 \\
\hline DVD & 34,5 & Jogado no rio e/ou igarapé & 3,4 \\
\hline Celular & 34,5 & Queimado & 1,1 \\
\hline Rádio & 28,7 & Fuma & \\
\hline Canoa & 14,9 & Sim & 13,8 \\
\hline Ventilador & 14,9 & Não & 86,2 \\
\hline Cozinha interna & & Ingere e/ou já ingeriu bebida alcoólica & \\
\hline Sim & 88,5 & Sim & 24,1 \\
\hline Não & 11,5 & Não & 75,9 \\
\hline
\end{tabular}

Tabela 2. Estado nutricional das crianças quilombolas de acordo com os índices E/I, P/I e IMC/I.

\begin{tabular}{cccc}
\hline \multirow{2}{*}{ Índice } & Classificação (\%) \\
\cline { 2 - 4 } E/I & 23,4 & Adequado & Acima \\
$\mathrm{P} / \mathrm{I}$ & 6,8 & 70,3 & 6,3 \\
$\mathrm{IMC} / \mathrm{I}$ & 4,6 & 88,9 & 4,3 \\
\hline
\end{tabular}

As análises estatísticas mostraram associação significativa entre crianças com déficit para o índice E/I e determinados aspectos de moradia e saneamento básico, como água para consumo proveniente de fontes impróprias, como rio e igarapés; além do destino do esgoto inadequado, como a céu aberto e/ou mato. Houve associação significativa entre as crianças com sobrepeso segundo o índice IMC/I e residências com melhor infraestrutura, como a presença de sanitário interno. O índice $\mathrm{P} / \mathrm{I}$ não apresentou associação com as variáveis independentes analisadas (Tabela 3). 
Tabela 3. Resíduos e níveis de confiança resultantes da aplicação da análise de correspondência às variáveis estado nutricional, segundo os indicadores de crescimento infantil versus perfil sócio demográfico.

\begin{tabular}{|c|c|c|c|c|c|}
\hline \multirow{2}{*}{ Índices } & \multirow{2}{*}{ Variáveis } & \multirow{2}{*}{ Categorias } & \multicolumn{3}{|c|}{ Estado Nutricional } \\
\hline & & & Déficit & Adequado & Excesso $* * *$ \\
\hline \multirow[t]{40}{*}{$\mathrm{E} / \mathrm{I}$} & \multirow[t]{4}{*}{ Sexo } & \multirow[t]{2}{*}{ Feminino } & 0,27 & 0,34 & $-1,95$ \\
\hline & & & $(21,29)$ & $(26,47)$ & $(0,00)$ \\
\hline & & \multirow[t]{2}{*}{ Masculino } & $-0,48$ & $-0,60$ & 3,49 \\
\hline & & & $(0,00)$ & $(0,00)$ & $(99,95)^{*}$ \\
\hline & \multirow[t]{6}{*}{ Pessoas na residência } & \multirow[t]{2}{*}{$<4$} & $-1,01$ & 0,36 & $t$ \\
\hline & & & $(0,00)$ & $(28,35)$ & \\
\hline & & \multirow[t]{2}{*}{4} & 2,80 & $-1,01$ & \\
\hline & & & $(99,50)^{*}$ & $(0,00)$ & \\
\hline & & \multirow[t]{2}{*}{$>4$} & $-2,08$ & 0,75 & \\
\hline & & & $(0,00)$ & $(54,44)^{* *}$ & \\
\hline & \multirow[t]{8}{*}{ Água para consumo próprio } & \multirow[t]{2}{*}{ Rio e/ou igarapé } & $-1,83$ & 0,91 & $t$ \\
\hline & & & $(0,00)$ & $(63,71)^{* *}$ & \\
\hline & & \multirow[t]{2}{*}{ Poço aberto } & 0,10 & $-0,05$ & \\
\hline & & & $(8,16)$ & $(0,00)$ & \\
\hline & & \multirow[t]{2}{*}{ Poço fechado } & 1,33 & 0,66 & \\
\hline & & & $(0,00)$ & $(49,21)$ & \\
\hline & & \multirow[t]{2}{*}{ Outros } & 2,72 & 1,35 & \\
\hline & & & $(99,35)^{*}$ & $(0,00)$ & \\
\hline & \multirow[t]{8}{*}{ Água para uso doméstico } & \multirow[t]{2}{*}{ Rio e/ou igarapé } & $-1,81$ & 0,89 & t \\
\hline & & & $(0,00)$ & $(62,48)^{* *}$ & \\
\hline & & \multirow[t]{2}{*}{ Poço aberto } & 0,17 & 0,08 & \\
\hline & & & $(13,48)$ & $(0,00)$ & \\
\hline & & \multirow[t]{2}{*}{ Poço fechado } & 1,52 & 0,75 & \\
\hline & & & $(0,00)$ & $(54,37)^{* *}$ & \\
\hline & & Encanamento & 2,61 & $-1,28$ & \\
\hline & & & $(99,10)^{*}$ & $(0,00)$ & \\
\hline & Destino do esgoto doméstico & Rio e/ou igarapé & $-1,33$ & 0,66 & $t$ \\
\hline & & & $(0,00)$ & $(49,17)$ & \\
\hline & & Céu aberto & 2,33 & $-1,15$ & \\
\hline & & & $(98,00)^{*}$ & $(0,00)$ & \\
\hline & & Fossa & 1,25 & 0,62 & \\
\hline & & & $(0,00)$ & $(46,55)$ & \\
\hline & Destino do esgoto do banheiro & Rio e/ou igarapé & $-1,62$ & 0,72 & $t$ \\
\hline & & & $(0,00)$ & $(52,94) * *$ & \\
\hline & & Céu aberto & 1,17 & $-0,52$ & \\
\hline & & & $(75,99)^{*}$ & $(0,00)$ & \\
\hline & & Fossa & 1,62 & 0,72 & \\
\hline & & & $(0,00)$ & $(52,94)^{* *}$ & \\
\hline & & Outros & 2,63 & $-1,17$ & \\
\hline & & & $(99,16)^{*}$ & $(0,00)$ & \\
\hline $\mathrm{P} / \mathrm{I}$ & Cozinha interna & Não & $-1,74$ & 0,92 & t \\
\hline & & & $(0,00)$ & $(63,99)$ & \\
\hline & & Sim & 0,72 & 0,38 & \\
\hline & & & $(52,54)$ & $(0,00)$ & \\
\hline $\mathrm{IMC} / \mathbf{I}$ & Sanitário interno & Não & + & $-0,36$ & 1,13 \\
\hline & & & & $(0,00)$ & $(74,18)^{*}$ \\
\hline & & Sim & & 0,54 & $-1,68$ \\
\hline & & & & $(41,04)$ & $(0,00)$ \\
\hline
\end{tabular}

* Probabilidades fortemente significativas. ** Probabilidades moderadamente significativas. ${ }^{* * *}$ Crianças classificadas com excesso segundo E/I também são consideradas adequadas. † Não foi possível aplicar a AC devido não haver o número mínimo de observações necessárias. 


\section{DISCUSSÃO}

Este estudo revelou que as condições socioeconômicas das famílias quilombolas investigadas eram precárias, marcadas pela baixa escolaridade dos adultos, baixa renda familiar, alta dependência de programas de redistribuição de renda (Bolsa Família) e núcleos familiares com até 5 pessoas (embora esta constituição seja menor do que no passado para os grupos populacionais tradicionais da Amazônia) ${ }^{31}$. Diversas pesquisas no nordeste e norte do país tem também demonstrado que nas comunidades quilombolas predominam a baixa escolaridade e baixa renda familiar ${ }^{32,33,34}$.

Em relação aos bens de consumo, foi possível identificar que as residências amostradas possuíam, em média, cinco itens, sendo os mais frequentes: televisão, geladeira e fogão. Algumas pesquisas sugerem que o aumento das despesas com aquisição de bens de consumo, principalmente em comunidades de baixa renda, se deve aos programas de transferência condicional de renda, como o Bolsa Família ${ }^{35,36}$, do qual a maioria dos participantes do presente estudo é beneficiária.

No Pará, o número de famílias quilombolas com acesso a eletricidade pública tem sido ampliado ${ }^{15}$. A Chamada Nutricional Quilombola indicou que mais de $75 \%$ das comunidades investigadas no país teve acesso considerado adequado a eletricidade ${ }^{15}$. No entanto, como centenas de outras populações quilombolas ${ }^{16}$, o acesso à iluminação pública ainda está ausente em grande parcela das comunidades analisadas, que continuam a depender, sobretudo, de pequenos geradores locais. Realidade diferente, por exemplo, das comunidades quilombolas mineiras estudadas por Silva ${ }^{35}$, nas quais a maior parte das famílias já tem acesso à energia elétrica.

Com relação ao saneamento ambiental, as condições de infraestrutura dos remanescentes de quilombos investigados foram consideradas precárias, pois a maioria das casas não possuía sistema de esgoto e nem fontes de água e tratamento adequado dos dejetos, situação similar à observada em outra pesquisa realizada com quilombolas das diversas regiões do Pará3 ${ }^{4}$. Esta é também a realidade de outros estados, como entre os remanescentes de quilombos do Vale do Mucuri, Minas Gerais ${ }^{37}$

Ao considerar os aspectos comportamentais, um estudo de base populacional, realizado com quilombolas do sudoeste da Bahia, mostrou que $54,1 \%$ deles nunca havia fumado ${ }^{32}$, corroborando parcialmente com os resultados deste estudo. Vale ressaltar que, em populações que residem distante das zonas urbanas, é mais comum o uso de cigarros feitos pelos próprios fumantes do que a utilização de cigarros e outros produtos industrializados ${ }^{38}$. No entanto, ainda há poucos estudos sobre esses hábitos entre populações rurais e quilombolas.

Em relação à ingestão de álcool, o hábito de consumir bebida alcoólica atinge $38,1 \%$ da população total do Brasil, sendo vários os fatores associados a isto, incluindo escolaridade, estado civil e condição socioeconômica ${ }^{39}$. Uma pesquisa realizada em comunidades quilombolas da Bahia mostrou que a maioria $(58,5 \%)$ relatou não ingerir bebida alcoólica regularmente ${ }^{40}$, perfil semelhante ao das populações quilombolas paraenses. No entanto, apesar dos quilombolas não relatarem nas entrevistas um padrão elevado de consumo de álcool, este é um fator de preocupação uma vez que as evidências observacionais nas comunidades mostraram-se contraditórias entre o que foi relatado nas entrevistas e o cotidiano comunitário.

Semelhante à situação de crianças ribeirinhas paraenses ${ }^{41}$ e daquelas que participaram da Chamada Nutricional Quilombola ${ }^{15}$, nas crianças analisadas observou-se elevada frequência de déficit estatural, indicativo de desnutrição crônica, principalmente entre aqueles que vivem em condições inadequadas de moradia e saneamento básico. Estes resultados corroboram com a pesquisa global recente em crianças menores de cinco anos de 64 países em desenvolvimento, que mostrou que os valores dos índices E/I e IMC/I no início da vida ( $<3$ anos) estão diretamente associados à situação socioeconômica das famílias ${ }^{42}$.

Além do déficit estatural, verificou-se também que as crianças quilombolas apresentaram elevada prevalência de baixo $\mathrm{P} / \mathrm{l}$, chegando próximo aos resultados médios nacionais da última Chamada Nutricional Quilombola ${ }^{15}$, na qual a porcentagem de crianças acometidas por baixo $\mathrm{P} / \mathrm{I}$ foi de $8,1 \%$, enquanto que no presente estudo foi de $6,8 \%$. Entretanto, as crianças quilombolas paraenses apresentaram uma prevalência de excesso de peso duas vezes maior que os observados na pesquisa nacional ${ }^{15}$.

A última Pesquisa de Orçamento Familiar, realizada em 2008-2009, mostrou também como estão dispostas as curvas de evolução do peso mediano segundo as idades das crianças e adolescentes brasileiros. Identificou-se que a curva de evolução do peso mediano ultrapassou o padrão esperado, independentemente da idade e do sexo. Para as crianças de até 5 anos de idade, em ambos os sexos, a diferença existe, mas ficou relativamente próxima ao padrão internacional. Essas diferenças para mais em relação ao padrão esperado para a evolução do peso, quando combinadas com as informações relativas ao padrão de crescimento, apontam na direção da elevação dos índices de excesso de peso nas crianças brasileiras ${ }^{43}$, o que já começa a ser observado, também, entre as crianças quilombolas $27,28,44$. 
Uma análise igualmente importante é a comparação das curvas de evolução do peso mediano em função do local de moradia das crianças, ou seja, a situação das medidas quando o foco está nas diferenças entre as áreas urbana e rural. É interessante verificar que as crianças brasileiras residentes em áreas urbanas já ultrapassam o padrão internacional de referência, independente do sexo, enquanto, em geral, as crianças das áreas rurais ainda não apresentam tal característica. No entanto, a curva de evolução do peso mediano das crianças rurais já se encontra quase que sobreposta à curva do padrão esperado ${ }^{43}$.

Neste estudo há prevalência de $11,1 \%$ de crianças com excesso de peso segundo o IMC/I, o que reforça o cenário epidemiológico de transição nutricional ocorrendo no Brasil, emergindo, inclusive em populações socialmente vulnerabilizadas, como os quilombolas, os ribeirinhos e os residentes em favelas ${ }^{17,35,45}$.

A falta de censos demográficos que englobem completamente as populações rurais, principalmente da região Norte, e os entraves à identificação dos territórios não permitem conhecer o tamanho e o número real dos grupos remanescentes de quilombos, dificultando inferências quanto à representatividade global da amostra estudada, o que representa uma limitação deste estudo, reforçando a importância da realização de estudos com este tipo de delineamento em outros grupos rurais vulnerabilizados que, apesar de serem parte expressiva da população no Brasil e, principalmente, na Amazônia, ainda são pouco representados nas pesquisas científicas.

Considerando que a amostra de crianças é potencialmente representativa do conjunto das populações remanescentes de quilombos na Amazônia, as análises estatísticas mostraram que o estado nutricional das crianças, principalmente em relação ao índice E/I, está associado a algumas características de moradia e saneamento básico. Concomitante a este cenário, o excesso de peso já atinge uma parcela relevante das crianças quilombolas, confirmando o processo de transição nutricional, com potenciais graves implicações para a saúde destas crianças no futuro ${ }^{46,47}$. No entanto, para os demais índices, não foi observada correlação significante. Os motivos para tais resultados não se mostram evidentes no momento, mas fatores como os cuidados parentais com a criança, o padrão de organização familiar, a histórica insegurança alimentar e nutricional, e o racismo vividos por estas populações, são fatores que precisam ser mais detalhadamente investigados.

\section{AGRADECIMENTOS}

Os autores agradecem às comunidades quilombolas, que participaram ativamente das pesquisas; ao CNPq, DECIT/
Ministério da Saúde, FAPESPA, UFPA, pelo apoio financeiro aos projetos; a Professora Mestre Priscila Matos de Pinho pela sua colaboração na revisão final deste trabalho e aos revisores da Revista Ciência e Saúde pelas suas contribuições valiosas.

\section{REFERÊNCIAS}

1. Lopes F. Raça, saúde e vulnerabilidades. In: Batista LE, Kalckmann S. Raça, etnia e saúde. São Paulo: Instituto de Saúde; 2003. p. 7-11.

2. Chor D, Lima CRA. Aspectos epidemiológicos das desigualdades raciais em saúde no Brasil. Cad Saúde Pública. 2005;21(5):1586-94. https://doi.org/10.1590/S0102-311X2005000500033

3. Brasil. Ministério da Saúde. Secretaria de Atenção à Saúde. Departamento de Atenção Básica. Coordenação-Geral da Política de Alimentação e Nutrição. Relatório sobre sistema de vigilância alimentar e nutricional: módulo de gestão do Programa Bolsa Família na Saúde no Estado do Pará. Brasília, DF; 2008.

4. Guerrero AFH. Situação nutricional de populações remanescentes de quilombos do município de Santarém - Pará, Brasil [tese]. Rio de Janeiro: Escola Nacional de Saúde Pública Sergio Arouca; 2010.

5. Comissão Pró-Índio de São Paulo [Internet]. [capturado 15 de dezembro de 2015]. Disponível em: http://www.quilombo.org.br/

6. Brasil. Ministério do Desenvolvimento Social e Combate à Fome Conselho Nacional de Assistência Social. Relatório GT Quilombola. Brasília, DF; 2007.

7. Volochko A, Batista LE. Saúde nos Quilombos. São Paulo: Instituto de Saúde; 2009.

8. Martorell R. Improved nutrition in the first 1000 days and adult human capital and health. Am J Hum Biol. 2017;29:1-12. https:// doi.org/10.1002/ajhb.22952

9. Macrae F. A baby's first 1,000 days' determines their health prospects for life [Internet]. 2011 [capturado 29 de agosto de 2015]. Disponível em: http://www.dailymail.co.uk/health/article-2026482/ Babys-1-000-days-determine-health-prospects-life.html

10. Black RE, Victora CG, Walker SP, Bhutta ZA, Christian P, Onis M, Ezzati M, Grantham-McGregor S, Katz J, Martorell R, Uauy R. Maternal and child undernutrition and overweight in low-income and middle-income countries. Lancet. 2013;382(9890):427-51. https://doi.org/10.1016/S0140-6736(13)60937-X

11. Brasil. Ministério da Saúde. Cadernos de Atenção Básica. Saúde da criança: crescimento e desenvolvimento. Brasília, DF; 2012.

12. Leroy JL, Ruel M, Habicht JP, Frongillo EA. Linear growth deficit continues to accumulate beyond the first 1000 days in low- and middle-income countries: global evidence from 51 national surveys. J Nutr. 2014;144(9):1460-6. https://doi.org/10.3945/jn.114.191981

13. Martins VJB, Toledo Florêncio TMM, Grillo LP, Franco MCP, Martins PA, Clemente APG, Santos CDL, Vieira MFA, Sawaya AL. Longlasting effects of undernutrition. Int J Environ Res Public Health. 2011;8:1817-46. https://doi.org/10.3390/ijerph8061817

14. Brasil. Ministério da Saúde. Secretaria de Atenção à Saúde. Departamento de Atenção Básica. Orientações para a coleta e análise de dados antropométricos em serviços de saúde: Norma Técnica do Sistema de Vigilância Alimentar e Nutricional - SISVAN. Brasília, DF; 2011. 
15. Brasil. Ministério do Desenvolvimento Social e Combate a Fome. Secretaria de Avaliação e Gestão da Informação. Cadernos de Estudos Desenvolvimento Social em Debate. Políticas sociais e chamada nutricional quilombola: estudos sobre condições de vida nas comunidades e situação nutricional das crianças. Brasília, DF; 2008.

16. Oliveira ASJ. Quilombolas do Pará: condições de vulnerabilidade nas comunidades remanescentes de Quilombo. Assis: Triunfal; 2011.

17. Siqueira PP, Alves JGB, Figueiroa JN. Fatores associados ao excesso de peso em crianças de uma favela do Nordeste brasileiro. Rev Paul Ped. 2009;27(3):251-7. https://doi.org/10.1590/S0103-0582 2009000300004

18. Lopes PCS, Prado SRLA, Colombo P. Fatores de risco associados à obesidade e sobrepeso em crianças em idade escolar. Rev Bras Enferm. 2010;1-6. https://doi.org/10.1590/S0034-71672010000100012

19. Silva HP, Padez C, Moura EAF, Filgueiras LA. Social determinants of health among traditional Amazonian populations: obesity, hypertension and the epidemiologic transition. Ann Hum Biol. 2016; 43(4):371-81. https://doi.org/10.1080/03014460.2016.1197967

20. Ziol-Guest KM, Duncan GJ, Kalil A. Early childhood poverty and adult body mass index. Am J Public Health. 2009;99(3):527-32. https://doi.org/10.2105/AJPH.2007.130575

21. Alves JGB, Figueira F. Doenças do adulto com raízes na infância. Rio de Janeiro: Medbook Editora Científica; 2010.

22. Acevedo-Marin R, Castro EMR. No caminho de pedras de Abacatal: experiência social de grupos negros no Pará. 2aㅡ ed. Belém: NAEA/ UFPA; 2004.

23. Costa RC. "Como uma comunidade": formas associativas em Santo Antônio/PA: imbricações entre parentesco, gênero e identidade [dissertação]. Belém: Universidade Federal do Pará; 2008.

24. Borges WD. Prevalência da hipertensão arterial sistêmica e seus determinantes bioantropológicos em populações quilombolas da Amazônia [dissertação]. Belém: Universidade Federal do Pará; 2011.

25. Cavalcante IMS. Acesso e acessibilidade aos serviços de saúde em três quilombos na Amazônia Paraense: um olhar antropológico [dissertação]. Belém: Universidade Federal do Pará; 2011.

26. Melo FT, Silva HP. Doenças crônicas e os determinantes sociais da saúde em comunidades quilombolas do Pará, Amazônia, Brasil [Internet]. Rev ABPN. 2015;7(16):168-89 [capturado 27 de agosto de 2016]. Disponível em: http://abpnrevista.org.br/revista/index. php/revistaabpn1/article/view/103

27. Guimarães RCR. Condições socioeconômicas, demográficas e o crescimento de crianças quilombolas no Pará [dissertação]. Belém: Universidade Federal do Pará; 2016.

28. Guimarães RCR, Silva HP. Estado nutricional e crescimento de crianças quilombolas de diferentes comunidades do Estado do Pará. Amazônica. 2015;7(1):1-23. https://doi.org/10.18542/amazonica. v7i1.2156

29. World Health Organization. The WHO child growth standards. Geneva: WHO; 2006.

30. World Health Organization. WHO anthro plus: version 3.2.2 [Internet]. Geneva: WHO; 2011 [capturado 28 de outubro de 2009]. Disponivel em: http://www.who.int/childgrowth/software/en/
31. Silva HP. Aspectos demográficos e médico-epidemiológicos dos residentes na Floresta Nacional da Caxiuanã, Melgaço, Pará. Belém: Museu Paraense Emílio Goeldi; 2002.

32. Bezerra VM, Andrade ACS, César CC, Caiaffa WT. Domínios de atividade física em comunidades quilombolas do sudoeste da Bahia, Brasil: Estudo de base populacional. Cad Saúde Pública. 2015;31(3):1213-24. https://doi.org/10.1590/0102-311X 00056414

33. Araújo AS, Anjos DR, Silva RS, Santos MAS, Martins CM, Almeida RHC. Análise socioeconômica de agricultores da comunidade quilombola do Abacatal, Ananindeua, estado do Pará, Brasil. Biota Amaz. 2017;7(1):30-7. https://doi.org/10.18561/2179-5746/biota amazonia.v7n1p30-37

34. Pinho PM, Pamplona VMS, Ramos EMLS. Avaliação nutricional em quilombolas marajoaras. Belém: UFPA; 2013.

35. Silva AAM. A desigualdade de renda no Brasil está diminuindo? Cad Saúde Pública. 2015;31(6):1125-6. https://doi.org/10.1590/0102311XED010615

36. Piperata BA, McSweeny D, Murrieta RSS. Conditional cash transfers, food security and health: biocultural insights for poverty-alleviation policy from the Brazilian Amazon. Curr. Anthropol. 2016;57(6): 1-21. https://doi.org/10.1086/68891238

37. Silva EA. Territórios quilombolas no Vale do Mucuri: As comunidades remanescentes de quilombo de Teófilo Otoni/MG. Rev Inst Ciên Hum. 2012;7(8):19-33.

38. Pinto MT, Pichon-Riviere A, Bardach A. Estimativa da carga do tabagismo no Brasil: mortalidade, morbidade e custos. Cad Saúde Pública. 2015;31(6):1283-97. https://doi.org/10.1590/0102-311X 00192013

39. Moura EC, Malta DC. Consumo de bebidas alcoólicas na população adulta brasileira: características sócio-demográficas e tendência. Rev Bras Epidemiol. 2011;14(1):61-70. https://doi.org/10.1590/S1415790X2011000500007

40. Cardoso LGV, Melo APS, Cesar CC. Prevalência do consumo moderado e excessivo de álcool e fatores associados entre residentes de comunidades quilombolas de Vitória da Conquista, Bahia, Brasil. Ciênc Saúde Colet. 2015;20(3):809-20. https://doi. org/10.1590/1413-81232015203.12702014

41. Silva SA, Moura EC. Determinantes do estado de saúde de crianças ribeirinhas menores de dois anos de idade do Estado do Pará, Brasil: um estudo transversal. Cad Saúde Pública. 2010; 26(2):273-85. https://doi.org/10.1590/S0102-311X20100002 00007

42. Murasko JE. Height, BMI, and relative economic standing in children from developing countries. Am J Hum Biol. 2017;29(3). https://doi. org/10.1002/ajhb.22958

43. Instituto Brasileiro de Geografia e Estatística. Pesquisa de Orçamento Familiar - POF 2008/2009. Antropometria e estado nutricional de crianças, adolescentes e adultos no Brasil. Rio de Janeiro: IBGE; 2010.

44. Filgueiras LA. Crianças ribeirinhas e quilombolas da Amazônia: crescimento, determinantes sociais de saúde e políticas públicas [tese]. Belém: Universidade Federal do Pará; 2016.

45. Filgueiras LA, Silva HP. Condições ecológicas e de saúde da população da Floresta Nacional de Caxiuanã, Pará, Brasil: análises 
antropométricas preliminares. In: VI Seminário Brasileiro sobre Áreas Protegidas e Inclusão Social; II Encontro Latino Americano sobre Áreas Protegidas e Inclusão Social. Áreas protegidas e inclusão social: tendências e perspectivas. Belo Horizonte; 2013. v. 6. p. 1166-1176.

46. Martins IS, Marinho SP, Oliveira DC, Araújo EAC. Pobreza, desnutrição e obesidade: inter-relação de estados nutricionais de indivíduos de uma mesma família. Ciênc Saúde Colet. 2007;12(6):1553-65. https://doi.org/10.1590/S1413-81232007000600017

47. Netto-Oliveira ER, Oliveira AAB, Nakashima ATA, Rosaneli CF, Oliveira Filho A, Rechenchosky L, Moraes ACF. Sobrepeso e obesidade em crianças de diferentes níveis econômicos. Rev Bras Cineantropom Desempenho Hum. 2010;12(2):83-9. https://doi. org/10.5007/1980-0037.2010v12n2p83 